\title{
Identification of Potential Key Genes Involved in the Carotid Atherosclerosis
}

\author{
Youshi Meng ${ }^{1,2, *}$ \\ Chunli Zhang ${ }^{1,2, *}$ \\ Lucong Liang ${ }^{1,2, *}$ \\ Lei Wei ${ }^{1,2}$ \\ Hao Wang ${ }^{1,2}$ \\ Fengkun Zhou ${ }^{1,2}$ \\ Rongjie $\mathrm{Li}^{1,2}$ \\ Donghua Zou (1D ${ }^{1,2}$ \\ Xiaohua Huang (D) $^{3}$ \\ Jie $\mathrm{Liu}^{4}$
}

'Department of Neurology, The Fifth Affiliated Hospital of Guangxi Medical University, Nanning, Guangxi, 530022,

People's Republic of China; ${ }^{2}$ Department of Neurology, The First People's Hospital of Nanning, Nanning, Guangxi, 530022, People's Republic of China; ${ }^{3}$ Department of Neurology, The Affiliated Hospital of Youjiang Medical University for

Nationalities, Baise, Guangxi, 533000,

People's Republic of China; ${ }^{4}$ Department of Cardiology, The Fifth Affiliated Hospital of Guangxi Medical University, Nanning, Guangxi, 530022, People's Republic of China

*These authors contributed equally to this work
Correspondence: Xiaohua Huang Department of Neurology, The Affiliated Hospital of Youjiang Medical University for Nationalities, Baise, Guangxi, 533000, People's Republic of China

Email newxiaohua66@I63.com

Jie Liu

Department of Cardiology, The Fifth

Affiliated Hospital of Guangxi Medical

University, Nanning, Guangxi, 530022 .

People's Republic of China

Tel +8677/2636I89

Email gxnnliujie@I63.com
Purpose: Carotid atherosclerosis is a kind of systemic atherosclerosis in the carotid arteries. However, the efficiency of treatment is insufficient. Therefore, it is urgent to find therapeutic targets and deepen the understanding of carotid atherosclerosis.

Materials and Methods: In this study, we analyzed differentially expressed genes (DEGs) between atheroma plaque and macroscopically intact tissue (control samples). Furthermore, we performed Gene Ontology (GO) and Kyoto Encyclopedia of Gene and Genomes (KEGG) enrichment analysis based on the DEGs. Four methods were used to identify the hub genes in the protein-protein interaction networks of the DEGs. Furthermore, we also performed network module analysis to reveal carotid atherosclerosis-related gene modules and biological functions.

Results: The enrichment results showed that the biological functions were related to inflammation, immunity, chemokine and cell adhesion molecule, such as PIK-Akt signaling pathway, Rap1 signaling pathway, MAPK signaling pathway, NOD-like receptor signaling pathway and B cell receptor signaling pathway. In addition, we screened the hub genes. A total of 16 up-regulated genes (C3AR1, CCR1, CCR2, CD33, CD53, CXCL10, CXCL8, CXCR4, CYBB, FCER1G, FPR2, ITGAL, ITGAM, ITGAX, ITGB2, and LILRB2) were identified as hub genes. A total of 5 gene modules were obtained. We found that biological functions obtained for each cluster were mostly related to immunity, chemokines and cell adhesion molecules.

Conclusion: The present study identified key DEGs in atheroma plaque compared with control samples. The key genes involved in the development of carotid atherosclerosis may provide valuable therapeutic targets for carotid atherosclerosis.

Keywords: identification of key genes, carotid atherosclerosis, cluster analysis

\section{Introduction}

Carotid atherosclerosis is the manifestation of systemic atherosclerosis in the carotid arteries. ${ }^{1}$ It usually occurs in adolescence and gradually worsens with age. It is currently believed to be closely related to the occurrence of ischemic stroke in the elderly. ${ }^{2}$ In the early stage of carotid atherosclerosis, it first manifests as intimamedia thickening, and then gradually forms atherosclerotic plaques. ${ }^{3}$ This cause corresponding hemodynamic changes, which in turn leads to ischemic cerebrovascular events. Contrary to the steady or declining trend of most disease, the incidence of atherosclerosis has been shown to increase in both men and women. ${ }^{4}$ In the past decade, the number of available treatments for carotid atherosclerosis has increased. In particular, immunotherapy and targeted therapy have improved the prospects of treatment. However, the efficiency of treatment is insufficient. ${ }^{5,6}$ 
Therefore, it is urgent to find therapeutic targets and deepen the understanding of carotid atherosclerosis.

Some studies have shown that the abnormal expression of some genes is closely related to the development of carotid atherosclerosis. S100A12, a calcium binding protein involved in inflammatory signaling, was also shown to be elevated in patients bearing carotid atherosclerotic lesions. ${ }^{7}$ Serum levels of TNF $\alpha$ and L-selectin, a lectin type adhesion molecule expressed on leukocytes surface, were shown to associate with larger plaque size. ${ }^{8}$ A previous study showed that there is an association between histological features of carotid plaque instability and serum levels of circulating matrix metalloproteinase (MMP)-1, MMP-7, tissue inhibitor of matrix protease (TIMP)-1, and IL- $8 .{ }^{9}$ But the molecule mechanism of carotid atherosclerosis has not been fully elucidated. Therefore, it is important to deepen the understanding for the relationship between carotid atherosclerosis and abnormal changes in genes.

In this study, we obtained the differentially expressed genes (DEGs) between atheroma plaque and control samples. We further explored the biological functions of DEGs and constructed a protein-protein interaction (PPI) network. In addition, we obtained 16 hub genes. And we established the carotid atherosclerosis developmentrelated network modules. In summary, we identified of hub genes involved in the development of carotid atherosclerosis. The study may help to provide guideline for the treatment of carotid atherosclerosis.

\section{Materials and Methods}

\section{Data Collection and Processing}

The expression profiling data of hypertensive patients in our study was derived from one published dataset (GSE43292) ${ }^{10}$ available in Gene expression omnibus (GEO) database. The GSE43292 dataset based on the GPL6244 platform contains 32 atheroma plaque and 32 macroscopically intact tissue (control samples) from 32 hypertensive patients. The dataset was profiled using the Affymetrix Human Gene 1.0 ST Array (Santa Clara, CA) platform. The justRMA method in the affy package $^{11}$ was applied to normalize the raw data of the dataset. If one gene corresponded to multiple probes, the average expression value of these probes was considered to be the expression value of the gene. The workflow of this study is shown in Figure 1.

\section{Analysis of Differentially Expressed Genes (DEGs) for Atheroma Plaque and Control Samples}

The limma package ${ }^{12}$ was used to analyze differentially expressed genes (DEGs) between atheroma plaque and control samples. The DEGs with $\mathrm{P}$ value (adjusted false positive rate) $<0.05$ and $\mid \log 2$ fold change $(\mathrm{FC}) \mid>0.5$ were considered significant.

\section{Enrichment Analysis}

The clusterProfiler package ${ }^{13}$ in $\mathrm{R}$ was used to functionally analyze the enriched pathways of the DEGs, including Gene Ontology (GO) terms and Kyoto Encyclopedia of Genes and Genomes (KEGG) pathways. P value $<0.05$ was considered significant. Gene set enrichment analysis (GSEA) was performed using GSEA java software $^{13}$ against the reference gene sets c2.cp.kegg. v6.2. symbols.gmt and c5.bp.v7.0.entrez.gmt, which were downloaded from the Molecular Signature Database (http://software.broadinstitute.org/gsea/index. jsp). ${ }^{13}$ The nominal (NOM) $\mathrm{P}$ value $<0.05$ and false discovery ratio $(\mathrm{FDR})<0.25$ were considered statistically significant.

\section{Protein-Protein Interaction (PPI)}

To obtain insights into the interactions among DEGs associated with carotid atherosclerosis. A PPI network was constructed using the Search Tool for the Retrieval of interacting Genes (STRING), ${ }^{14}$ a database of known and predicted protein interactions. An interaction with score $>990$ was included in our present study. The PPI network was visualized by Cytoscape 3.8.2 software (http://www.cytoscape.org/). ${ }^{15}$

\section{Hub Genes Selection and Analysis}

CytoHubba is a common tool for analyzing PPI networks. ${ }^{16}$ The hub genes were selected using the cytoHubba plug-in of Cytoscape software. Those overlap genes in the top 50 genes, from the four ranking methods, were defined as hub genes. The four ranking methods include Degree, density of maximum neighborhood component (DMNC), maximal clique centrality (MCC), maximum neighborhood component (MNC). 


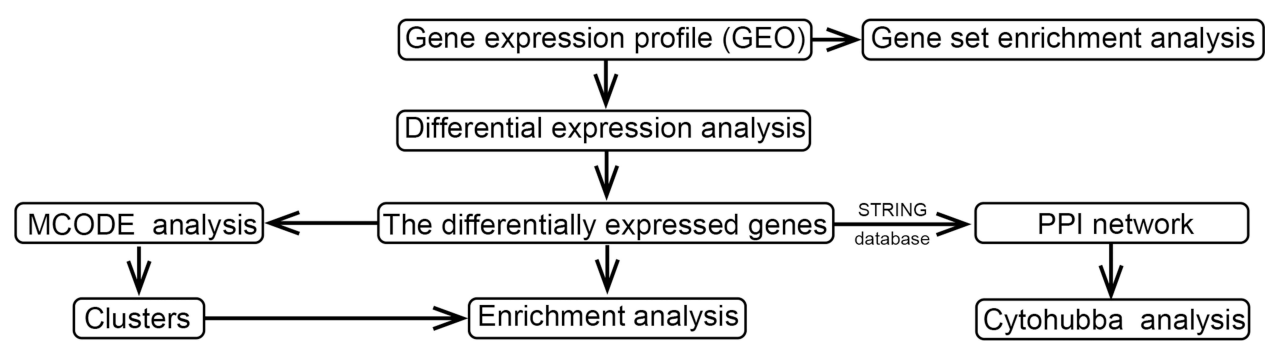

Figure I The workflow of this study.

Abbreviation: GEO, gene expression omnibus.

\section{Establishment of the Carotid Atherosclerosis-Related Network Modules}

We used the MCODE plug-in in the Cytoscape software ${ }^{17}$ to screen the modules concealed in the carotid atherosclerosis occurrence-related network with the following criteria: Max. depth $=100, \mathrm{~K}$-Core $=7$, mode score cutoff $=0.2$, and degree cutoff $=2$. Likewise, the functional specification of the identified module was determined with the clusterProfiler package as mentioned above. A P value (adjusted by Benjamini \& Hochberg method) $<0.05$ was considered statistically significant.

\section{Statistical Analysis}

All analyses were performed using R (version 4.0.2, http:// www.r-project.org/). We analyzed the expression levels of genes in the atheroma plaque and control samples using unpaired $t$-tests provided by limma package. Unless otherwise stated, we considered $\mathrm{P}<0.05$ to be statistically significant.

\section{Results \\ Identification of DEGs Associated with the Progression of Carotid Atherosclerosis}

A total of 1328 DEGs, including 749 up-regulated DEGs and 579 down-regulated DEGs, were identified in atheroma plaque compared with control samples (Figure 2A). In addition, we found that the expression patterns of these DEGs may distinguish atheroma plaque from control samples (Figure 2B)

\section{KEGG and GO Enrichment Analyses of DEGs}

To analyze the biological classification of DEGs, functional and pathway enrichment analyses were performed. As shown in Figure 3A, changes in biological processes (BPs) of DEGs were significantly enriched in the physical activity of immune cells (neutrophil, leukocyte, T cell, lymphocyte and phagocyte). GO analysis result showed that cellar component (CC) and molecular function (MF)
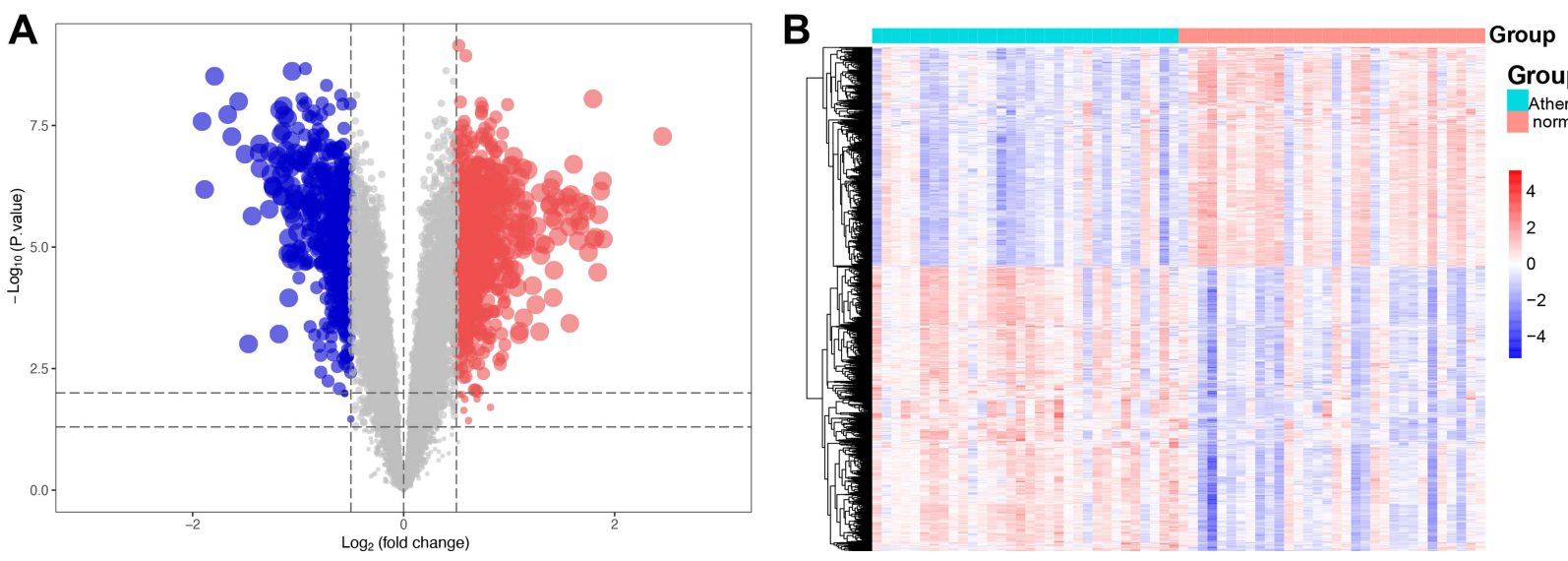

Figure 2 The differentially expressed genes (DEGs) for carotid atherosclerosis and control samples. (A) A volcano plot of the for carotid atherosclerosis and control samples. Red indicated upregulated genes and blue indicated downregulated genes. The gray area showed the gene expression below the threshold criteria. (B) The heatmap of the DEGs.

Abbreviation: DEGs, differentially expressed genes. 


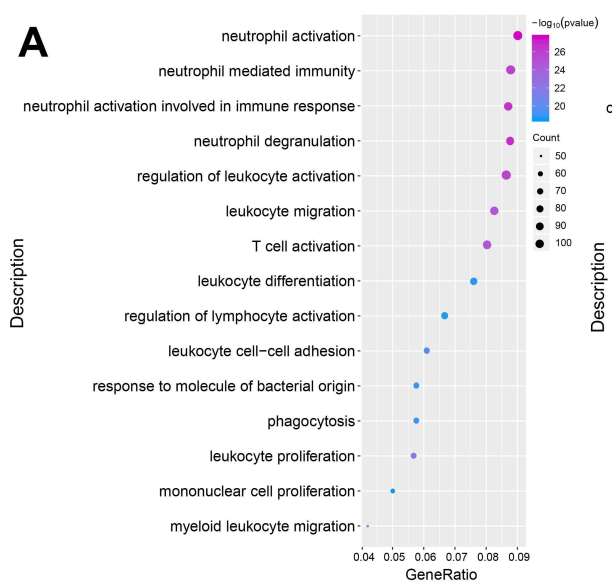

D $_{\text {Cytokine-cytokine receptor interaction }}$

PI3K-Akt signaling pathway

Chemokine signaling pathway

Rap1 signaling pathway

Cell adhesion molecules

Phagosome

음

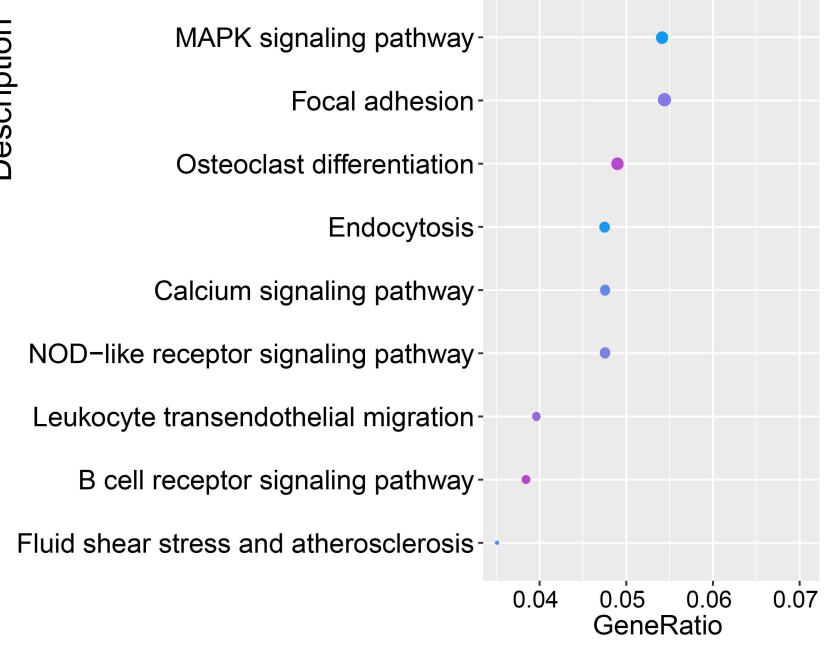

B extracelluar matrix

collagen-containing extracellular matrix-

secretory granule membrane

side of membrane

membrane region

membrane microdomain
membrane raft

receptor complex

tertiary granule

vacuolar lumen

specific granule

specific granule membrane-

lysosomal lumen

tertiary granule membrane $\begin{array}{lllll}0.02 & 0.03 & 0.04 & 0.05 & 0.06 \\ \text { GeneRatio } & & \end{array}$

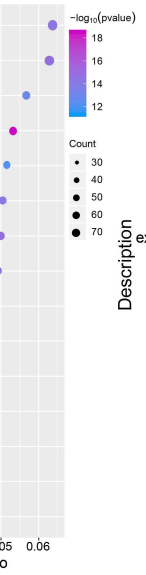

C cell adhesion molecule binding.

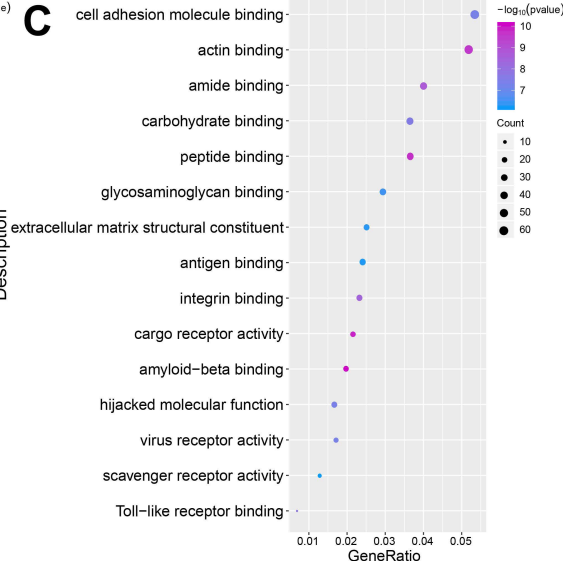

E

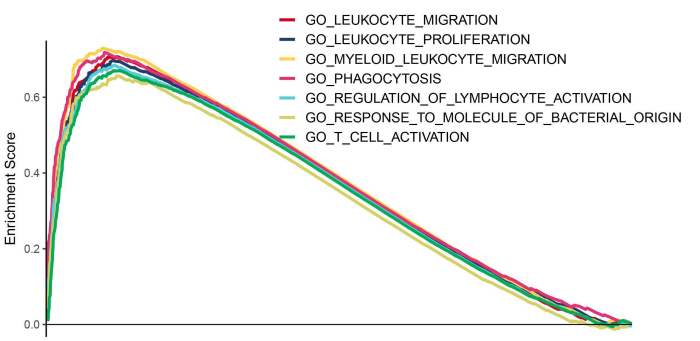

$\mathbf{F}$
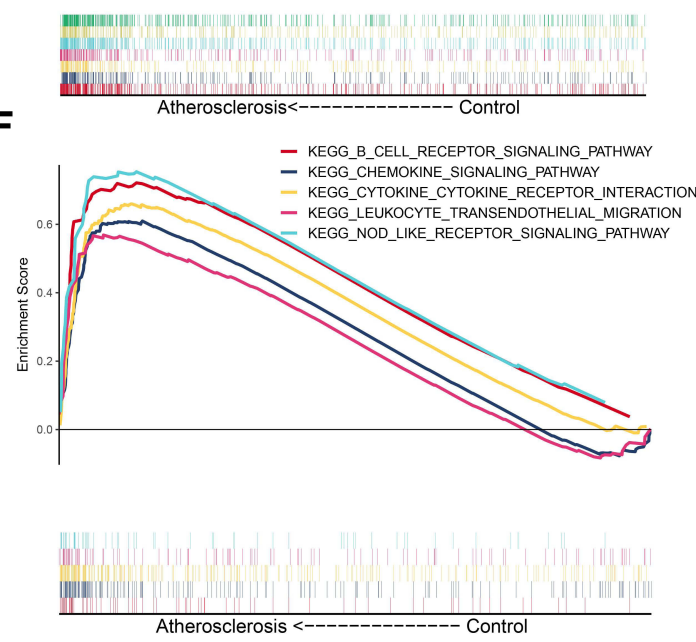

Figure 3 The biological functions of differentially expressed genes (DEGs). (A) Cellular component, (B) biological process, (C) molecular function and (D) KEGG pathway analysis. The gene set enrichment analysis (GSEA) for (E) GO and (F) KEGG for carotid atherosclerosis and control samples.

Abbreviations: GO, gene ontology; KEGG, Kyoto encyclopedia genes and genome.

were significantly enriched in "extracellular matrix", "plasma membrane protein complex", "collagencontaining extracellular matrix", "cell adhesion molecule binding" "actin binding" and "amide binding" (Figure 3B and C). The KEGG enrichment analysis result showed that some pathways were related to carotid atherosclerosis, such as PI3K-Akt signaling pathway, ${ }^{18}$ Chemokine signaling pathway, ${ }^{19}$ Cell adhesion molecules, ${ }^{20}$ MAPK signaling pathway, ${ }^{21}$ Calcium signaling pathway, ${ }^{22}$ NODlike receptor signaling pathway ${ }^{23}$ and Fluid shear stress and atherosclerosis ${ }^{24}$ (Figure 3D). Furthermore, we found that the change of BPs was significantly "leukocyte migration", "leukocyte proliferation", "myeloid leukocyte migration", "phagocytosis", "regulation of lymphocyte activation", "response to molecule of bacterial origin" and "T cell activation" in atheroma plaque compared with control samples based on GSEA result (Figure 3E). And the KEGG pathways, "B cell receptor signaling pathway", "chemokine signaling pathway", "cytokine-cytokine receptor interaction", "leukocyte 
trans endothelial migration" "NOD like receptor signaling pathway", were significantly enriched in atheroma plaque compared with control samples based on GSEA result (Figure 3F).

\section{PPI Network Construction}

To obtain the interactions between the 1328 DEGs in the atheroma plaque compared with control samples, a PPI network was constructed using the STRING database and visualized by the Cytoscape software. As shown in Figure 4, the network included 147 nodes and 178 edges.

\section{Hub Genes Selection and Analysis}

Among the lists of the top 50 genes selected, respectively, by the four ranking methods (Figure 5A-D), 16 genes overlapped were identified (Figure 5E). And 16 hub genes (C3AR1, CCR1, CCR2, CD33, CD53, CXCL10, CXCL8, CXCR4, CYBB, FCER1G, FPR2, ITGAL, ITGAM, ITGAX, ITGB2, and LILRB2) were upregulated in carotid atherosclerosis tissue compared with control samples (Figure 5F).

\section{KEGG and GO Enrichment Analyses of Cluster Genes}

A total of 5 clusters were obtained after MCODE (Molecular Complex Detection) algorithm. In addition, the enrichment analysis result showed that changes in GO function of cluster 1 were significantly enriched in "neutrophil activation", "secretory granule membrane" and "G protein-coupled peptide receptor activity" (Figure 6A), while the changes in KEGG pathways were significantly enriched in "chemokine signaling pathway" and "cytokine-cytokine receptor interaction" (Figure 6B). The changes in GO function of cluster 2 were significantly enriched in "response to type I interferon", "defense response to other organism", "endocytic vesicle", "plasma membrane protein complex", "toll-like receptor activity", "protein tyrosine kinase activity" and "cytokine receptor binding" (Figure 7A), while the changes in KEGG pathways of that were significantly enriched in "Tuberculosis" (Figure 7B). The changes in GO function of cluster 3 were significantly enriched in "neutrophil activation", "azurophil granule lumen", "primary lysosome",

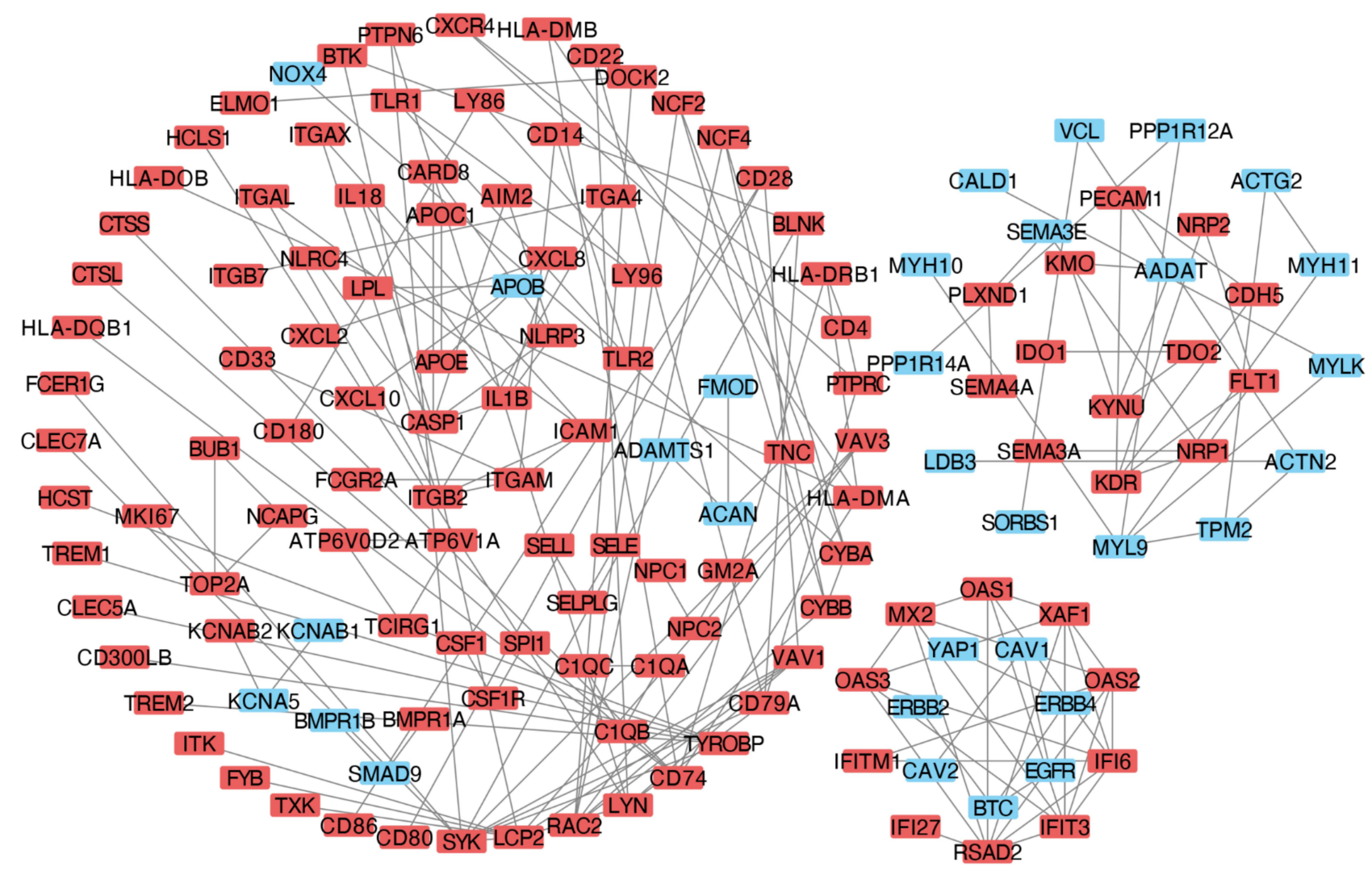

Figure 4 The PPI network. A graphic representation of the protein-protein network based on differentially expressed genes of carotid atherosclerosis and control samples. Red nodes indicated upregulated genes and light blue nodes indicated downregulated genes in the carotid atherosclerosis group. 

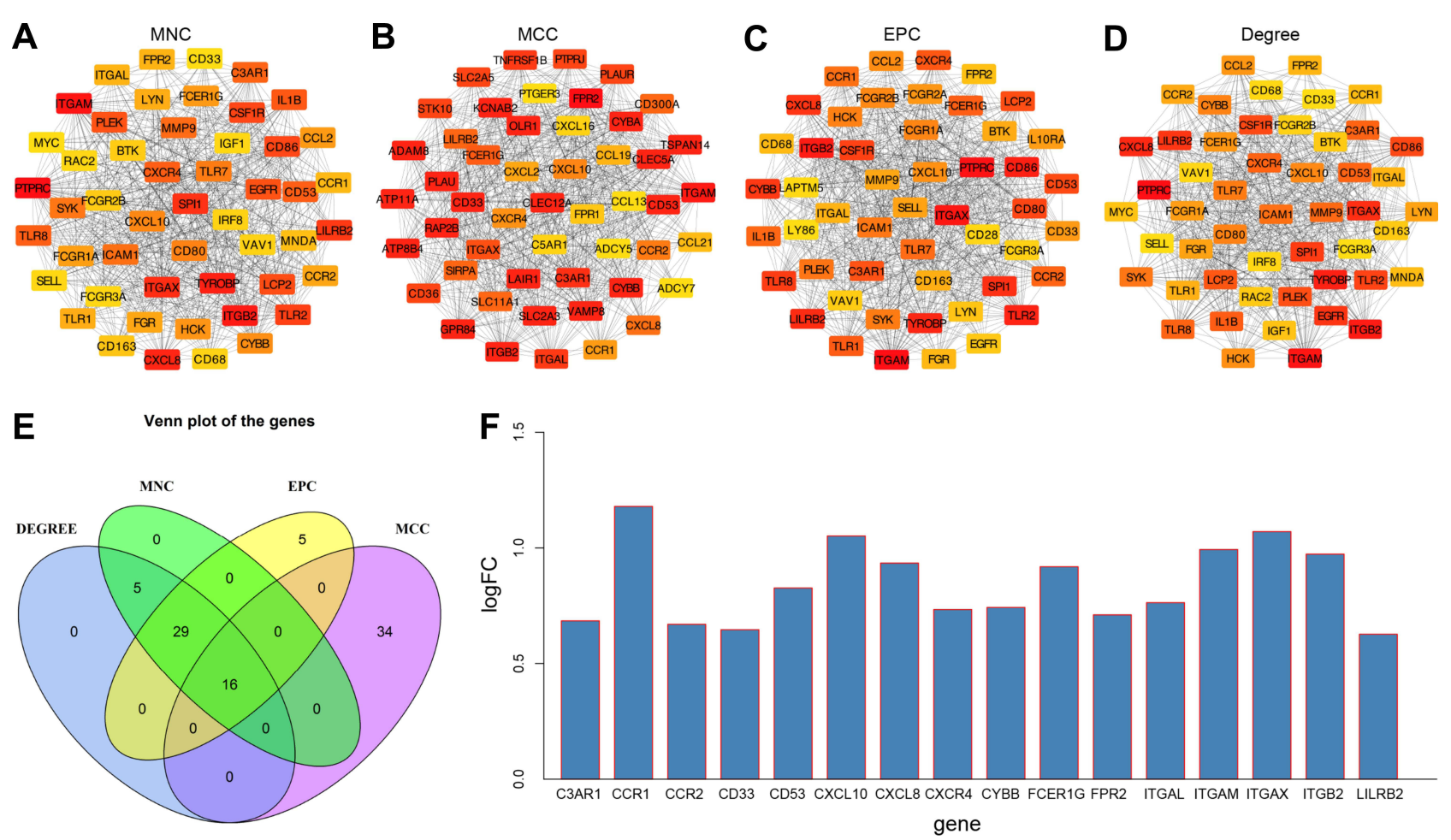

Figure 5 The selection and analysis of hub genes. Top 50 genes selected based the four ranking methods. (A) Degree, (B) DMNC, (C) MCC and (D) MNC. The darker the color of the node, the higher the score. DMNC, density of maximum neighborhood component; MCC, maximal clique centrality. MNC, maximum neighborhood component. (E) Venn plot. Venn plot of the overlapping genes for the top 50 genes selected based on the four ranking methods. Degree, DMNC, MNC, and MCC. (F) The expression levels for 16 hub genes in carotid atherosclerosis samples compared with control samples.

"azurophil granule", "vacuolar lumen", "secretory granule lumen", "vesicle lumen", "receptor ligand activity", "enzyme activator activity" and "receptor regulator activity" (Figure 8A), while the changes in KEGG pathways of that were significantly enriched in "lysosome" and "rRheumatoid arthritis" (Figure 8B). The changes in GO function of cluster 4 were significantly enriched in "regulation of leukocyte activation", "cytoplasmic vesicle lumen", "vesicle lumen", "ubiquitin-protein transferase activity" and "ubiquitin-like protein transferase activity" (Figure 9A), while the changes in KEGG pathways of that were significantly enriched in "PI3K-Akt signaling pathway", "human papillomavirus infection", "endocytosis", "focal adhesion" and "cell adhesion molecules" (Figure 9B). The changes in GO function of cluster 5 were significantly enriched in "muscle system process", "actin cytoskeleton" and "actin binding" (Figure 10A), while the changes in KEGG pathways of that were significantly enriched in "vascular smooth muscle contraction" (Figure 10B). These results indicated that the formation of carotid atherosclerosis is the result of a coordination disorder of multiple gene modules and various biological pathways.

\section{Discussion}

A study showed that about 57.79 million people of $30 \sim 70$ year-old in the world have atheroma plaque in 2020, an increase of $59.13 \%$ from $2000 .{ }^{25}$ However, the efficiency of treatment was low. To find potentially effective and therapeutic targets, there is an urgent requirement to explore the genes that lead to development of carotid atherosclerosis.

The present study utilized a relatively large sample dataset obtained from GEO. These data were analyzed to identify DEGs between carotid atherosclerosis and control samples. KEGG pathway analysis revealed with the 1328 DEGs mainly involved with PI3K-Akt signaling pathway, Chemokine signaling pathway, Cell adhesion molecules, MAPK signaling pathway, Calcium signaling pathway, NOD-like receptor signaling pathway and Fluid shear stress and atherosclerosis. It is well known that the inhibition of PI3K-Akt signaling pathway may reduce the vulnerability of atherosclerotic plaques. ${ }^{18}$ And chemokine CXCR4 may limit atherosclerosis by maintaining arterial integrity. ${ }^{19}$ Furthermore, geniposide may be against 

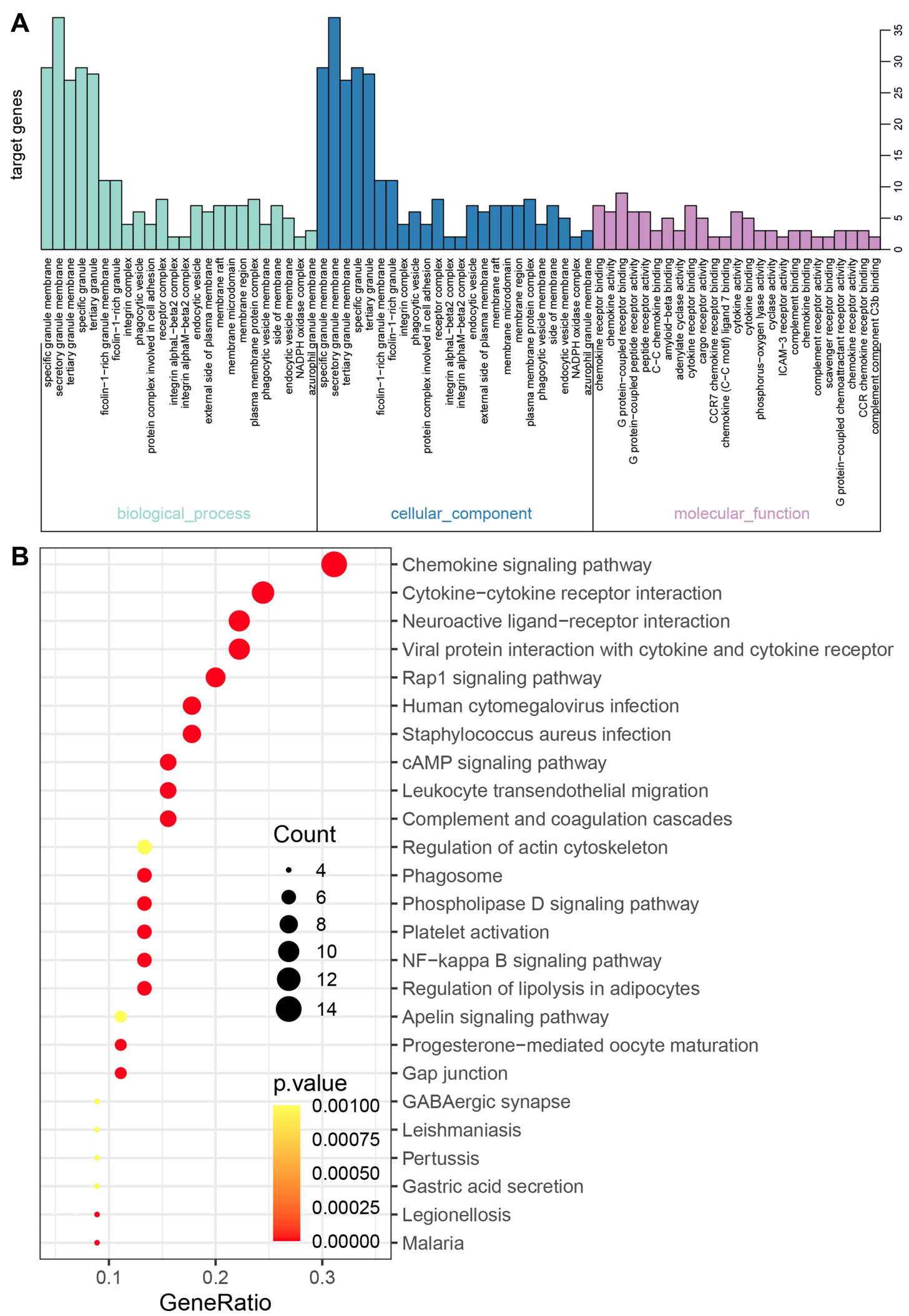

Figure 6 The GO and KEGG enrichment analysis for the genes in cluster I obtained based the MCODE method. (A) The biological processes and (B) KEGG pathways for genes in cluster $\mathrm{I}$. 


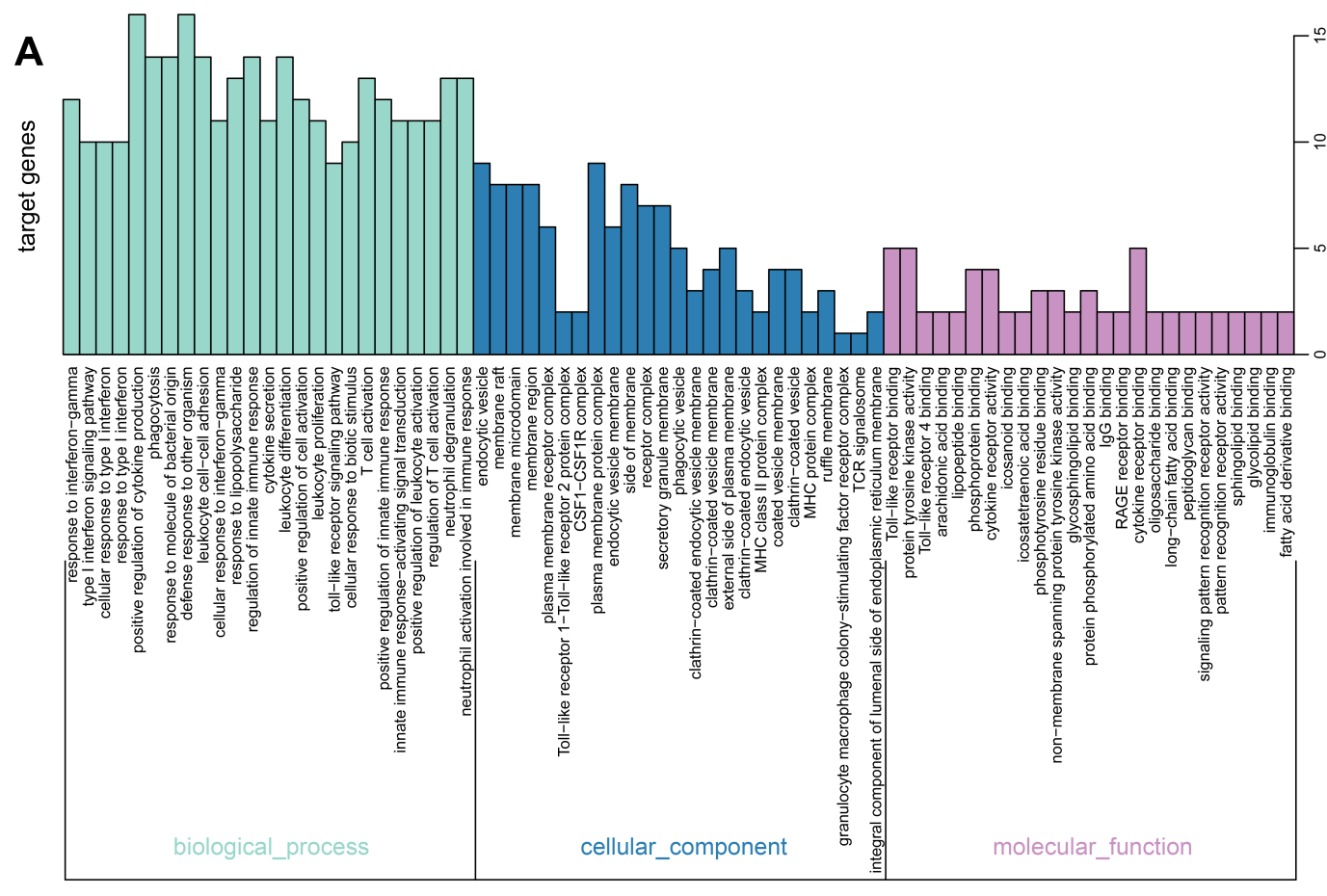

B

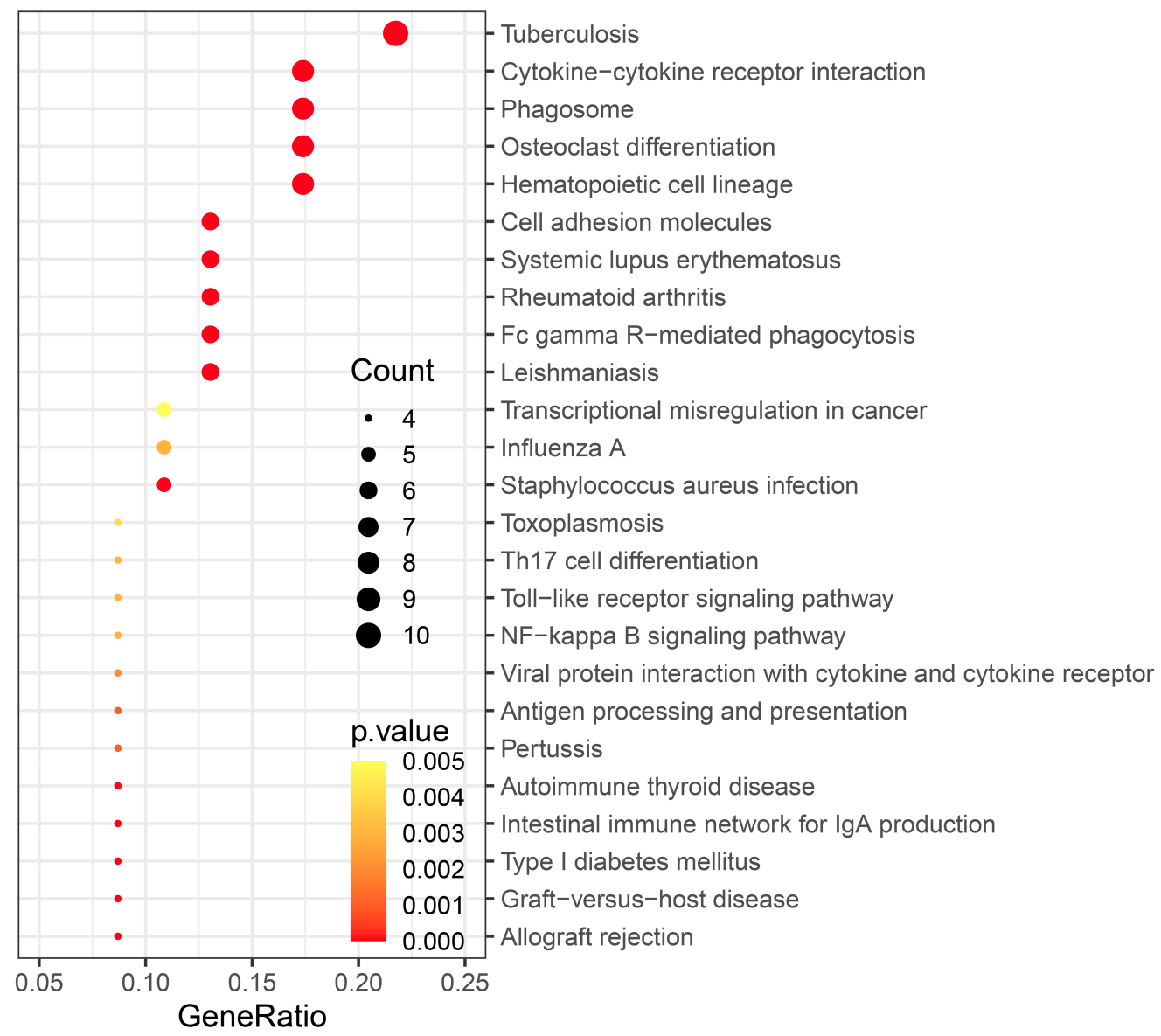

Figure 7 The GO and KEGG enrichment analysis for the genes in cluster 2 obtained based the MCODE method. (A) The biological processes and (B) KEGG pathways for genes in cluster 2. 

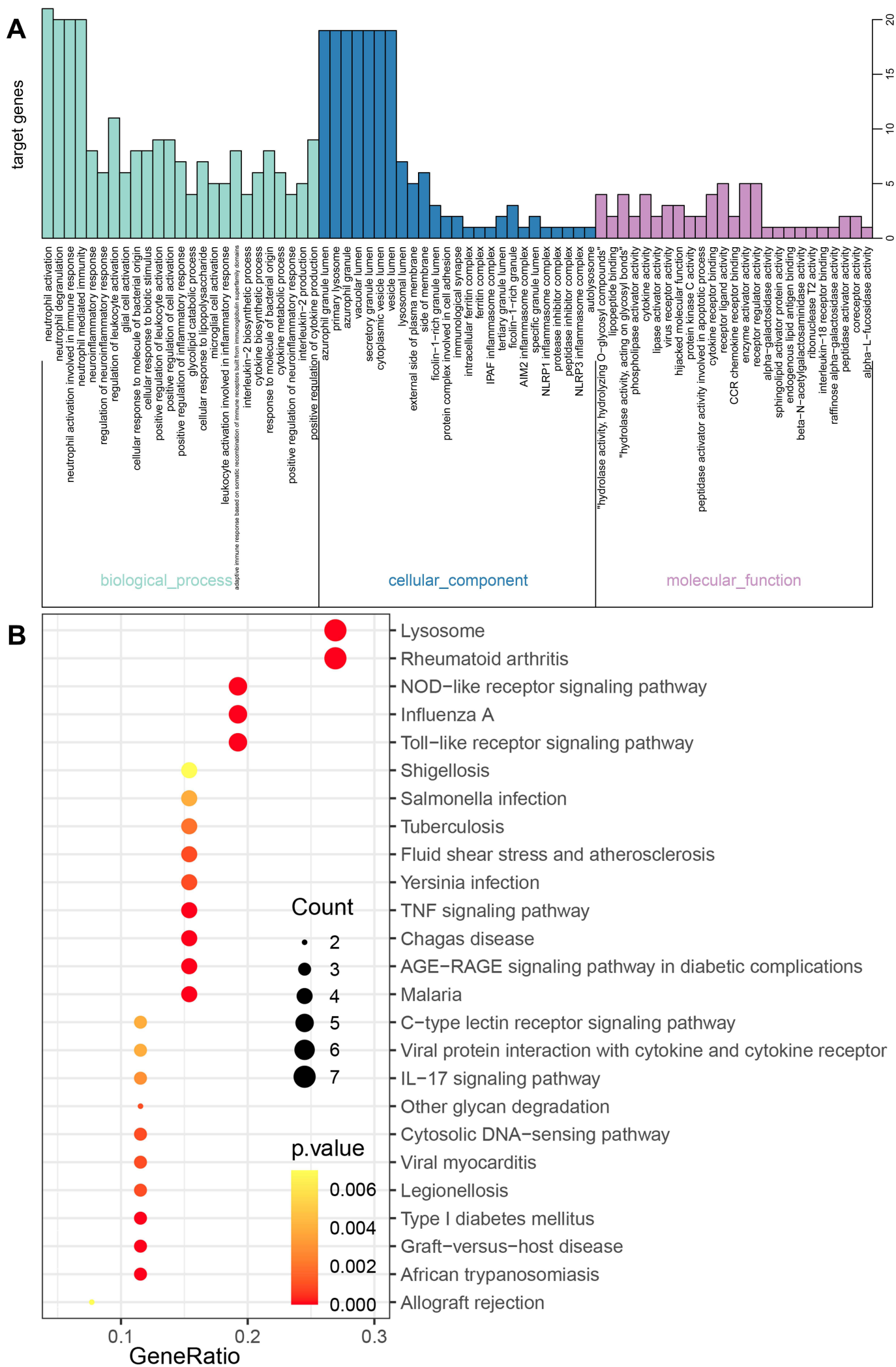

Figure 8 The GO and KEGG enrichment analysis for the genes in cluster 3 obtained based the MCODE method. (A) The biological processes and (B) KEGG pathways for genes in cluster 3 . 


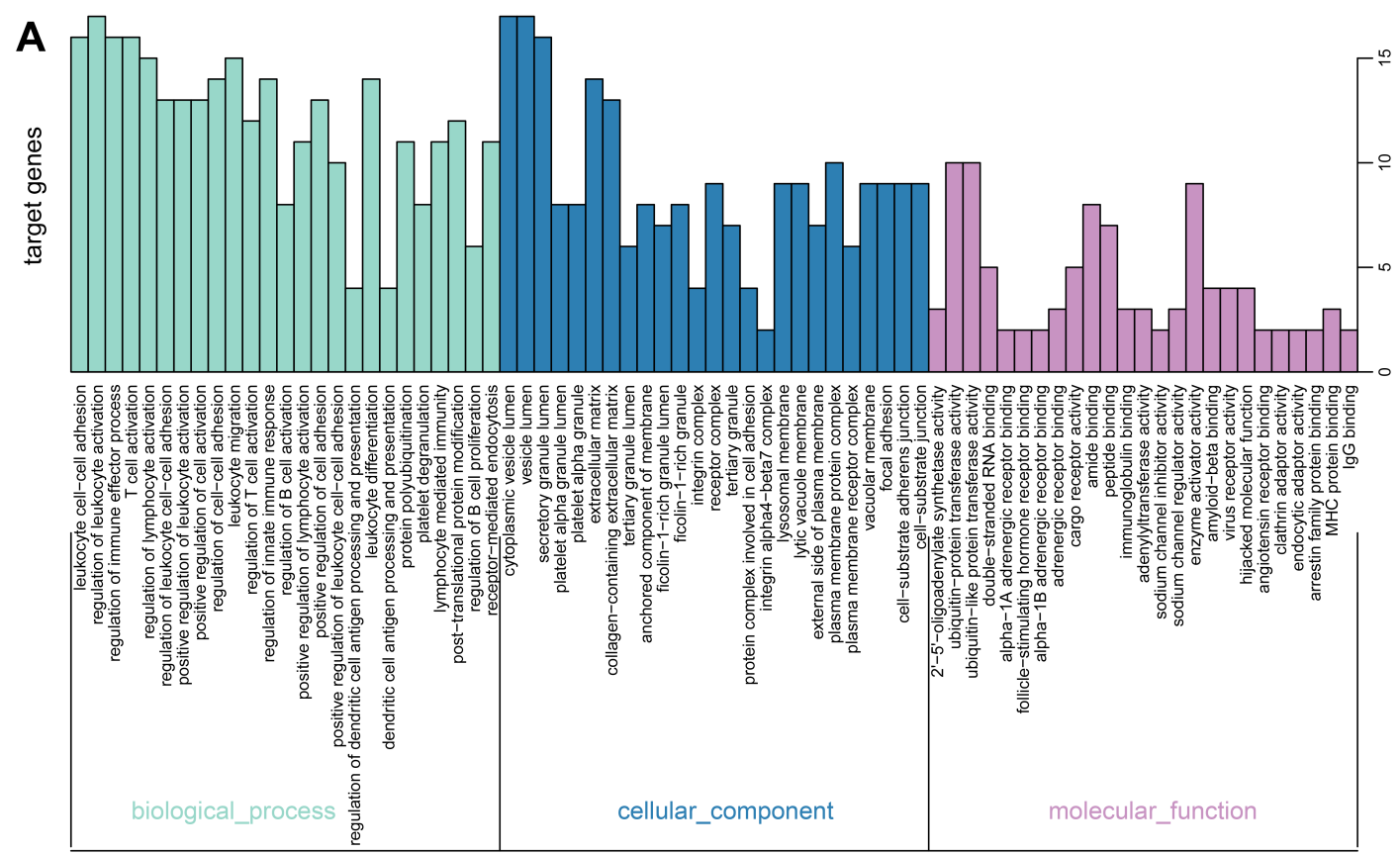

B

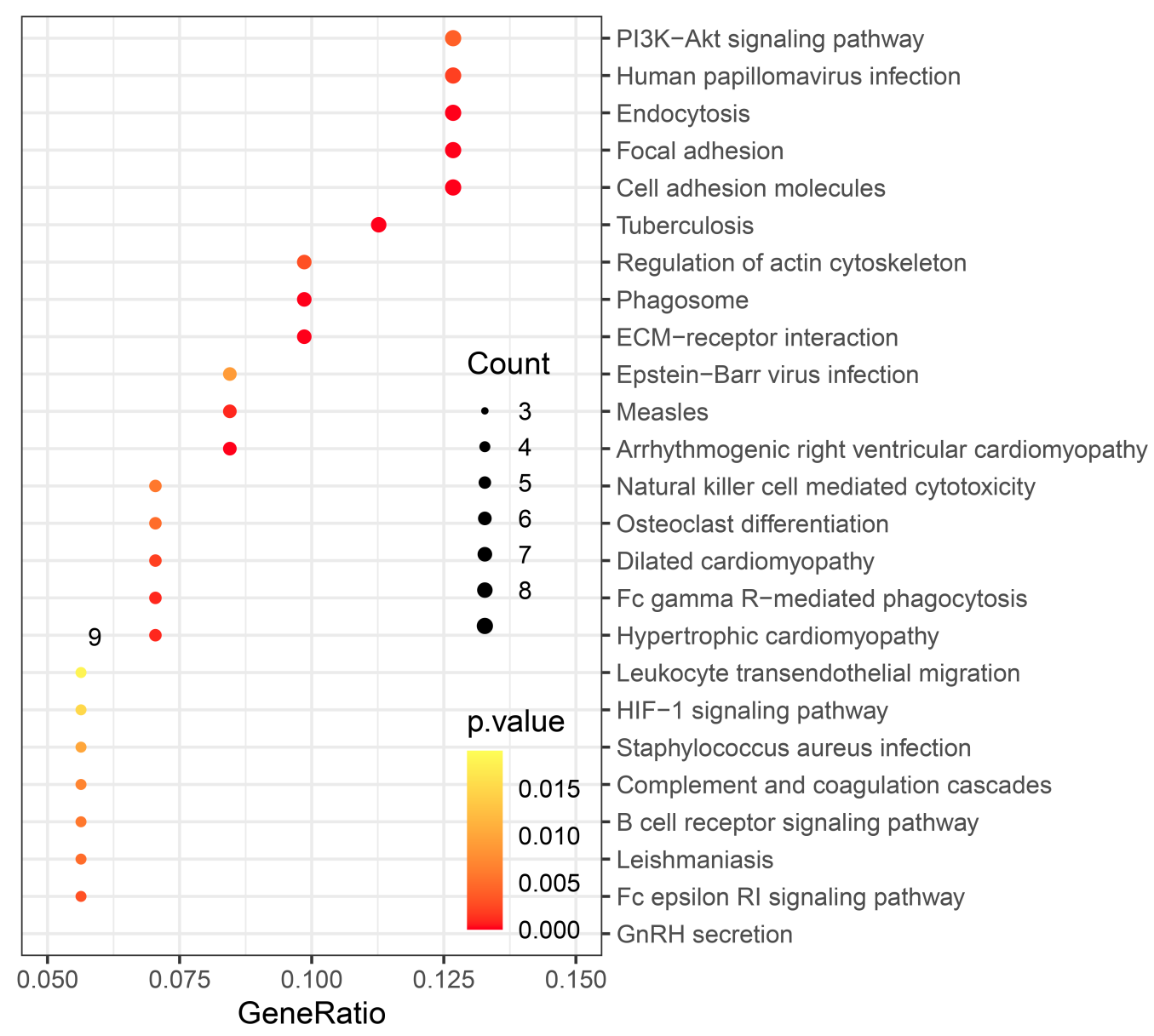

Figure 9 The GO and KEGG enrichment analysis for the genes in cluster 4 obtained based the MCODE method. (A) The biological processes and (B) KEGG pathways for genes in cluster 4. 


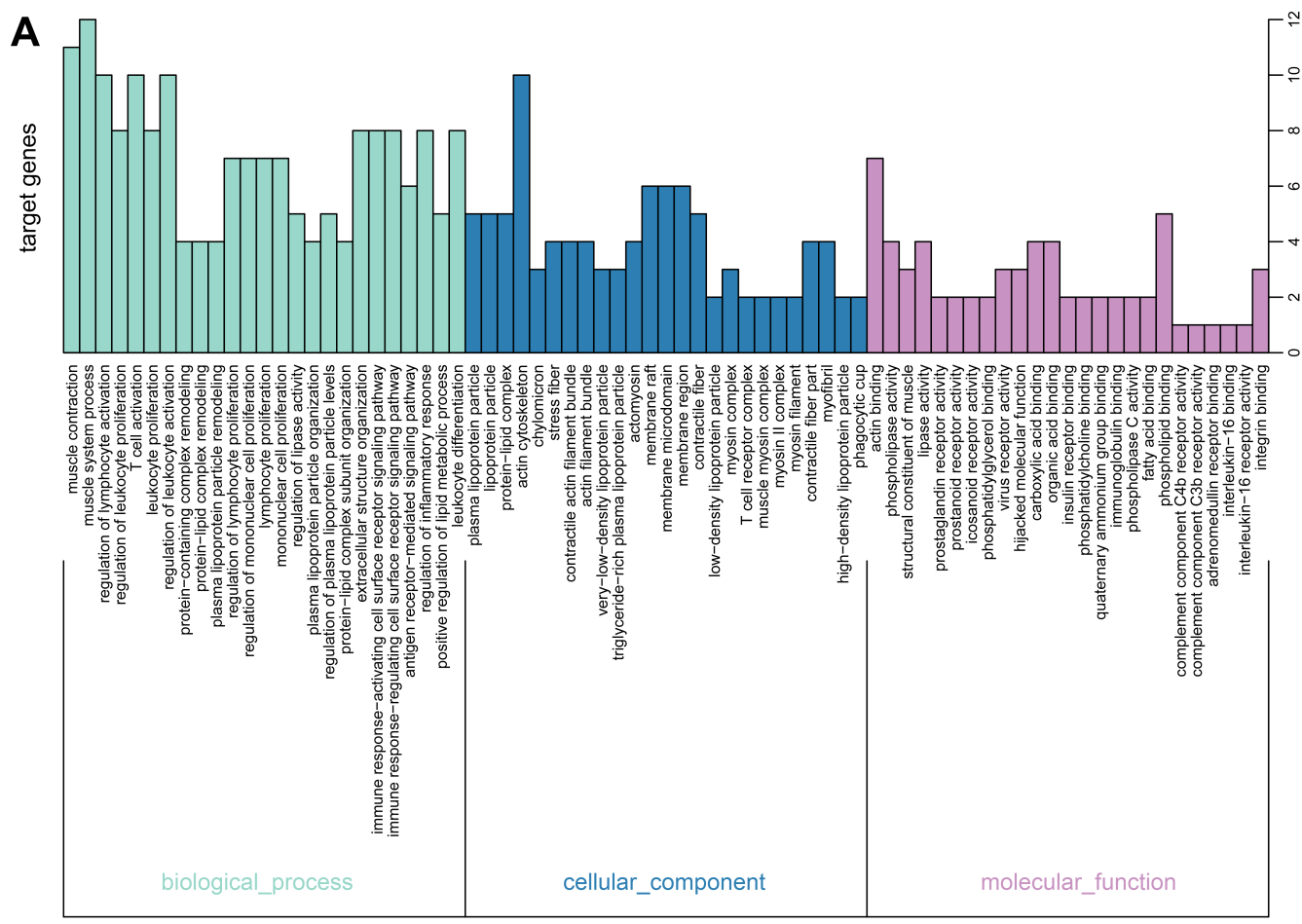

B

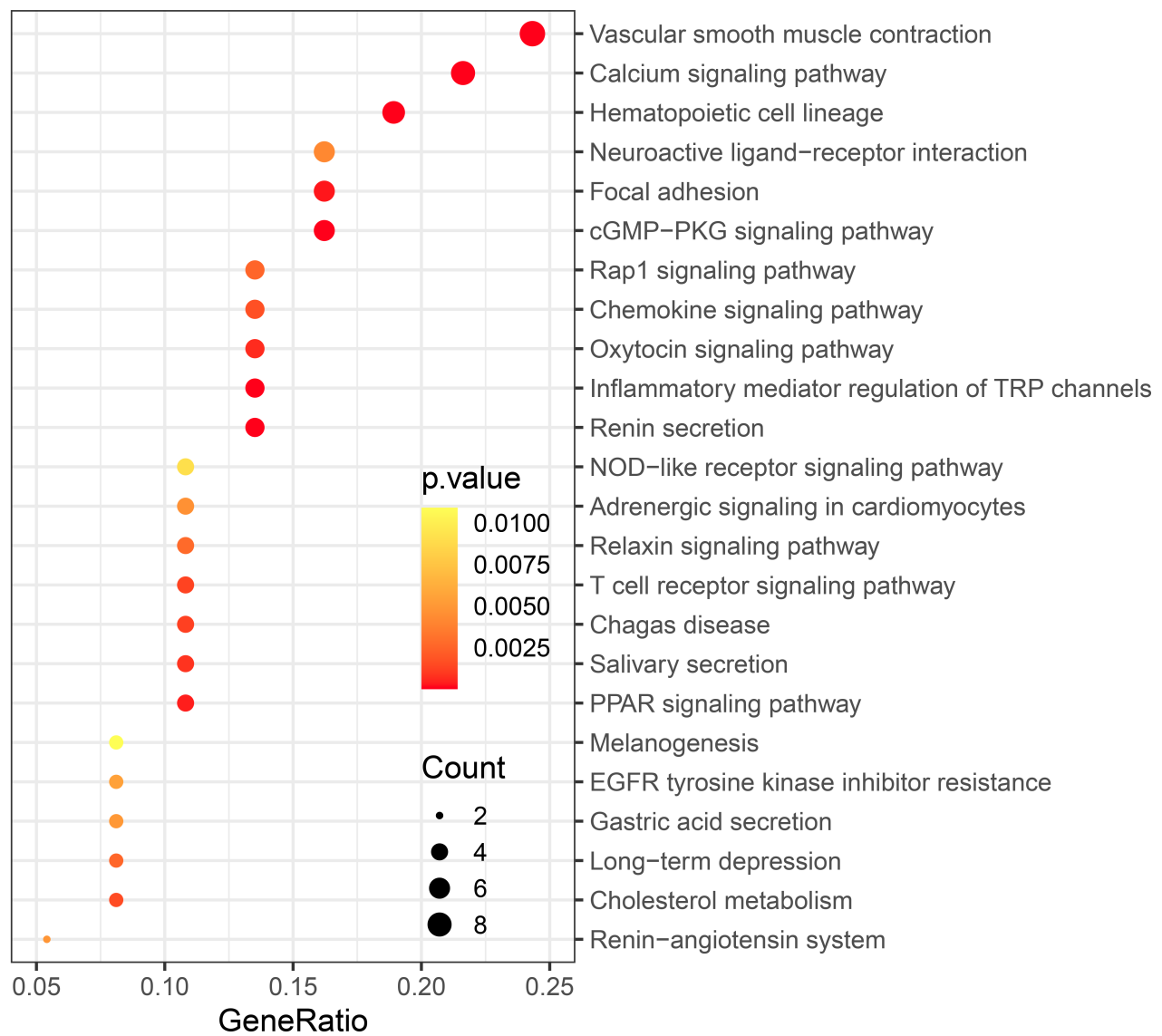

Figure 10 The GO and KEGG enrichment analysis for the genes in cluster 5 obtained based the MCODE method. (A) The biological processes and (B) KEGG pathways for genes in cluster 5 . 
atherosclerosis by inhibiting formation of foam cell and lowering reverse lipid transport via MAPK signaling pathway. ${ }^{21}$ Circulating cell adhesion molecules are correlated with ultrasound-based assessment of carotid atherosclerosis. ${ }^{20} \mathrm{GO}$ enrichment analysis revealed that DEGs were mainly associated with physical activity of immune cells (neutrophil, leukocyte, T cell, lymphocyte and phagocyte). The present results provide bioinformatics evidence for further research.

The 16 overlapping genes among the top 50 genes in the PPI network found using four ranking methods were selected. All 16 genes were upregulated in the carotid atherosclerosis. The result reveals that these genes may serve an important role in the progression of carotid atherosclerosis. And prior the present study, few studies have addressed the gaps in the molecular mechanisms that lead to carotid atherosclerosis development. The genes (C3AR1, CCR1, CCR2, CXCL10, CXCL8, CXCR4) enriched for chemokine-related genes. The genes (ITGAL, ITGAM, ITGAX, ITGB2) enriched for cell adhesion-related molecules. The genes (CD33, CD53, CYBB, FCER1G, FPR2, LILRB2) enriched for immunity-related genes. In carotid plaques, $\mathrm{C} 3 \mathrm{aR} 1^{26}$ and FCER $1 G^{27}$ is higher compared to control arteries. CCR1 alters the immuno-inflammatory response in atherosclerosis. ${ }^{28}$ Anti-CXCL10 treatment in atherosclerosis susceptible mice results in a change into a more stable lesion phenotype. ${ }^{29}$ The progression of carotid atherosclerosis is related to CD53. ${ }^{30}$ In the present study, the aim was to focus on DEGs between atheroma plaque and control samples. However, due to lack of experimental validation, it is not clear whether these genes are causal or merely markers. Furthermore, we established of the carotid atherosclerosis development-related network modules based on the MCODE method. A total of 5 clusters were obtained. Furthermore, the enrichment analysis results of each cluster genes showed that biological functions obtained were mostly related to immunity, chemokines and cell adhesion molecules. But there are some differences.

A total of 5 clusters were obtained after MCODE algorithm. And the enrichment analysis result showed that the biological function were associated with carotid atherosclerosis. Interestingly, NF-kb, a crucial transcriptional factor, which controls the transcription of many genes with an established role in atherosclerosis, such as cell proliferation, regulators of apoptosis, acute phase proteins, adhesion molecules, chemokines and cytokines. ${ }^{31}$ Toll-like receptors (TLRs), key players in innate immunity, are upregulated in atherosclerotic lesions. ${ }^{32}$ Atherosclerosis is an inflammatory disease associated with the activation of innate immune TLRs and nucleotide-binding oligomerization domaincontaining protein (NOD)-like receptor pathways. ${ }^{33}$ In addition, a previous study showed that a large amount of iron is deposited in the lysosomes of foam cells in early atherosclerotic lesions. ${ }^{34}$ In summary, we thought the 5 clusters were closely related to carotid atherosclerosis.

There are a few important limitations to the present study. Firstly, the study is only based on the bioinformatics analysis, lack of experimental validation. Secondly, the sample size in this study is not very large. We hope that we can expand the sample size included in the further. Finally, our study is based on a retrospective dataset. We hope that we can verify the findings of this study in a prospective cohort.

\section{Conclusion}

In conclusion, the present study identified key DEGs in atheroma plaque compared with control samples. The key genes involved in the development of carotid atherosclerosis may provide valuable therapeutic targets for carotid atherosclerosis.

\section{Data Sharing Statement}

Data were downloaded from the Gene Expression Omnibus (GEO) database (https://www.ncbi.nlm.nih.gov/ geo/).

\section{Acknowledgments}

This study was supported by the Project of Qingxiu District of Nanning Scientific Research and Technology Development Plan (2019039 and 2020058), the Scientific Research Project of Guangxi Health Commission (Z20200212), the High-Level Medical Expert Training Program of Guangxi “139” Plan Funding (G201903049), and Guangxi medical and health key discipline construction project (Department of Cardiology and Department of Emergency Medicine).

\section{Disclosure}

The authors report no conflicts of interest related to this work.

\section{References}

1. Stary HC, Chandler AB, Dinsmore RE, et al. A definition of advanced types of atherosclerotic lesions and a histological classification of atherosclerosis. A report from the Committee on Vascular Lesions of the Council on Arteriosclerosis, American Heart Association. Circulation. 1995;92(5):1355-1374. doi:10.1161/01.cir.92.5.1355 
2. Martinez E, Martorell J, Riambau V. Review of serum biomarkers in carotid atherosclerosis. J Vasc Surg. 2020;71(1):329-341. doi:10.1016/j.jvs.2019.04.488

3. Chen WH, Jin W, Lyu PY, et al. Carotid atherosclerosis and cognitive impairment in nonstroke patients. Chin Med $J$ (Engl). 2017;130 (19):2375-2379. doi:10.4103/0366-6999.215331

4. Forgo B, Medda E, Hernyes A, Szalontai L, Tarnoki DL, Tarnoki AD. Carotid artery atherosclerosis: a review on heritability and genetics. Twin Res Hum Genet. 2018;21(5):333-346. doi:10.1017/thg.2018.45

5. Harle LM, Plichart M. [Carotid atherosclerosis and dementia]. Geriatr Psychol Neuropsychiatr Vieil. 2015;13(3):309-316. French. doi:10.1684/pnv.2015.0554

6. Vigen T, Ihle-Hansen H, Lyngbakken MN, et al. Carotid atherosclerosis is associated with middle cerebral artery pulsatility index. $J$ Neuroimaging. 2020;30(2):233-239. doi:10.1111/jon.12684

7. Abbas A, Aukrust P, Dahl TB, et al. High levels of S100A12 are associated with recent plaque symptomatology in patients with carotid atherosclerosis. Stroke. 2012;43(5):1347-1353. doi:10.1161/ STROKEAHA.111.642256

8. Andersson J, Sundstrom J, Kurland L, et al. The carotid artery plaque size and echogenicity are related to different cardiovascular risk factors in the elderly: the Prospective Investigation of the Vasculature in Uppsala Seniors (PIVUS) study. Lipids. 2009;44 (5):397-403. doi:10.1007/s11745-009-3281-y

9. Pelisek J, Rudelius M, Zepper P, et al. Multiple biological predictors for vulnerable carotid lesions. Cerebrovasc Dis. 2009;28(6):601-610. doi: $10.1159 / 000247605$

10. Ayari H, Bricca G. Identification of two genes potentially associated in iron-heme homeostasis in human carotid plaque using microarray analysis. J Biosci. 2013;38(2):311-315. doi:10.1007/s12038-0139310-2

11. Gautier L, Cope L, Bolstad BM, Irizarry RA. Affy-analysis of Affymetrix GeneChip data at the probe level. Bioinformatics. 2004;20(3):307-315. doi:10.1093/bioinformatics/btg405

12. Yu J, Wu X, Huang K, et al. Bioinformatics identification of lncRNA biomarkers associated with the progression of esophageal squamous cell carcinoma. Mol Med Rep. 2019;19(6):5309-5320. doi:10.3892/ mmr.2019.10213

13. Yu G, Wang LG, Han Y, He QY. clusterProfiler: an R package for comparing biological themes among gene clusters. OMICS. 2012;16 (5):284-287. doi:10.1089/omi.2011.0118

14. Szklarczyk D, Gable AL, Lyon D, et al. STRING v11: protein-protein association networks with increased coverage, supporting functional discovery in genome-wide experimental datasets. Nucleic Acids Res. 2019;47(D1):D607-D613. doi:10.1093/nar/gky1131

15. Otasek D, Morris JH, Boucas J, Pico AR, Demchak B. Cytoscape automation: empowering workflow-based network analysis. Genome Biol. 2019;20(1):185. doi:10.1186/s13059-019-1758-4

16. Chin $\mathrm{CH}$, Chen SH, Wu HH, Ho CW, Ko MT, Lin CY. cytoHubba: identifying hub objects and sub-networks from complex interactome. BMC Syst Biol. 2014;8(Suppl 4):S11. doi:10.1186/1752-0509-8-S4$\mathrm{S} 11$

17. Bader GD, Hogue CW. An automated method for finding molecular complexes in large protein interaction networks. BMC Bioinform. 2003;4:2. doi:10.1186/1471-2105-4-2

18. Wang N, Zhang X, Ma Z, et al. Combination of tanshinone IIA and astragaloside IV attenuate atherosclerotic plaque vulnerability in ApoE(-/-) mice by activating PI3K/AKT signaling and suppressing TRL4/NF-kappaB signaling. Biomed Pharmacother. 2020;123:109729. doi:10.1016/j.biopha.2019.109729
19. Doring Y, Noels H, van der Vorst EPC, et al. Vascular CXCR4 limits atherosclerosis by maintaining arterial integrity: evidence from mouse and human studies. Circulation. 2017;136(4):388-403. doi:10.1161/CIRCULATIONAHA.117.027646

20. Rohde LE, Lee RT, Rivero J, et al. Circulating cell adhesion molecules are correlated with ultrasound-based assessment of carotid atherosclerosis. Arterioscler Thromb Vasc Biol. 1998;18 (11):1765-1770. doi:10.1161/01.atv.18.11.1765

21. Shen D, Zhao D, Yang X, Zhang J, He H, Yu C. Geniposide against atherosclerosis by inhibiting the formation of foam cell and lowering reverse lipid transport via p38/MAPK signaling pathways. Eur J Pharmacol. 2019;864:172728. doi:10.1016/j.ejphar.2019.172728

22. Zavaczki E, Gall T, Zarjou A, et al. Ferryl hemoglobin inhibits osteoclastic differentiation of macrophages in hemorrhaged atherosclerotic plaques. Oxid Med Cell Longev. 2020;2020:3721383. doi:10.1155/2020/3721383

23. Shi X, Xie WL, Kong WW, Chen D, Qu P. Expression of the NLRP3 inflammasome in carotid atherosclerosis. J Stroke Cerebrovasc Dis. 2015;24(11):2455-2466.

doi:10.1016/j. jstrokecerebrovasdis.2015.03.024

24. Chen Z, Qin H, Liu J, et al. Characteristics of wall shear stress and pressure of intracranial atherosclerosis analyzed by a computational fluid dynamics model: a pilot study. Front Neurol. 2019;10:1372. doi:10.3389/fneur.2019.01372

25. Song P, Xia W, Zhu Y, et al. Prevalence of carotid atherosclerosis and carotid plaque in Chinese adults: a systematic review and meta-regression analysis. Atherosclerosis. 2018;276:67-73. doi:10.1016/j.atherosclerosis.2018.07.020

26. Niyonzima N, Bakke SS, Gregersen I, et al. Cholesterol crystals use complement to increase NLRP3 signaling pathways in coronary and carotid atherosclerosis. EBioMedicine. 2020;60:102985. doi:10.1016/ j.ebiom.2020.102985

27. Centa M, Jin H, Hofste L, et al. Germinal center-derived antibodies promote atherosclerosis plaque size and stability. Circulation. 2019;139(21):2466-2482.

doi:10.1161/ CIRCULATIONAHA.118.038534

28. Potteaux S, Combadiere C, Esposito B, et al. Chemokine receptor CCR1 disruption in bone marrow cells enhances atherosclerotic lesion development and inflammation in mice. Mol Med. 2005;11 (1-12):16-20. doi:10.2119/2005-00028.Potteaux

29. Segers D, Lipton JA, Leenen PJ, et al. Atherosclerotic plaque stability is affected by the chemokine CXCL10 in both mice and humans. Int J Inflam. 2011;2011:936109. doi:10.4061/2011/936109

30. Zhao B, Wang D, Liu Y, et al. Six-gene signature associated with immune cells in the progression of atherosclerosis discovered by comprehensive bioinformatics analyses. Cardiovasc Ther. 2020;2020:1230513. doi:10.1155/2020/1230513

31. de Winther MP, Kanters E, Kraal G, Hofker MH. Nuclear factor kappaB signaling in atherogenesis. Arterioscler Thromb Vasc Biol. 2005;25(5):904-914. doi:10.1161/01.ATV.0000160340.72641.87

32. Monaco C, Gregan SM, Navin TJ, Foxwell BM, Davies AH, Feldmann M. Toll-like receptor-2 mediates inflammation and matrix degradation in human atherosclerosis. Circulation. 2009;120 (24):2462-2469. doi:10.1161/CIRCULATIONAHA.109.851881

33. Tang G, Duan F, Li W, et al. Metformin inhibited Nod-like receptor protein 3 inflammasomes activation and suppressed diabetes-accelerated atherosclerosis in apoE(-/-) mice. Biomed Pharmacother. 2019;119:109410. doi:10.1016/j.biopha.2019.109410

34. Jerome WG. Lysosomes, cholesterol and atherosclerosis. Clin Lipidol. 2010;5(6):853-865. doi:10.2217/clp.10.70 


\section{Publish your work in this journal}

Clinical Interventions in Aging is an international, peer-reviewed journal focusing on evidence-based reports on the value or lack thereof of treatments intended to prevent or delay the onset of maladaptive correlates of aging in human beings. This journal is indexed on PubMed Central, MedLine, CAS, Scopus and the Elsevier
Bibliographic databases. The manuscript management system is completely online and includes a very quick and fair peer-review system, which is all easy to use. Visit http://www.dovepress.com/ testimonials.php to read real quotes from published authors. 